ADDENDUM

\title{
A lex naturalis delineates components of a human-specific, adrenal androgen-dependent, p53-mediated 'kill switch' tumor suppression mechanism
}

\author{
Jonathan Wesley Nyce \\ ACGT Biotechnology, Collegeville, Pennsylvania, USA
}

Correspondence should be addressed to J W Nyce: nyce.jonathan@acgt.us

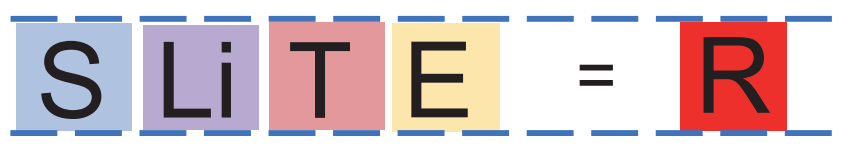

Figure 3

A normalized lex naturalis equation, representing a snapshot of a species with the dependent variables adult body size (S), lifespan (Li), speciesspecific mechanism of tumor suppression ( $T$ ), and carcinogen exposure $(E)$ in equilibrium to maintain lifetime cancer risk (R), at a value of about $4 \%$. We have previously defined a singularity as the original transformed, tumor-competent cell, while it is still in its single cell state. T is the probability of successful extinction of singularities by a particular species-specific tumor suppression mechanism, and hence its value is represented as a reciprocal, 1/T. $R$ is a measure of the probability of developing cancer at some time during the lifespan, and hence its value is also represented as a reciprocal, $1 / R$. These facts enable algebraic manipulation of the equation, e.g., $\mathrm{Li}=\mathrm{T} / \mathrm{SER}$. 